\title{
Quasi-Static Electrical Model for Magnetoresistive Current Sensors
}

\author{
A. Roldán ${ }^{1}$, C. Reig ${ }^{2}$, J.B. Roldán ${ }^{1}$, A. Cano-Abellán ${ }^{2}$, D. Ramírez ${ }^{2}$ \\ ${ }^{1}$ Department of Electronics and Computer Technology \\ Universidad de Granada \\ Av. Severo Ochoa, s/n - Granada, 18071 Granada (Spain) \\ Phone (Fax) number: +34 958244010 (43230), e-mail: amroldan@ugr.es, jbroldan@ugr.es \\ ${ }^{2}$ Department of Electronic Engineering \\ Universitat de València \\ Av. Dr. Moliner, 50 - Burjassot, 46100 València (Spain) \\ Phone (Fax) number: +34 963544023 (44353), e-mail: candid.reig@uv.es, aurelio.cano@uv.es, ramirez@uv.es
}

\begin{abstract}
The general use of magnetoresistive current sensors is limited due to the lack of electrical models, among other issues. In this paper, we report on a Verilog-A based quasi-static electrical model, focusing on the parameter extraction procedure. The accuracy of the model developed has been checked by comparing with experimental measurements for different measuring current values.
\end{abstract}

\section{Key words}

Verilog-A, magnetoresistive current sensor, GMR, AMR

\section{Introduction}

Traditionally, electrical current has been measured with shunt resistances, coils and solid state sensors [1]. The Ohm's law is in the basis of the first method and variations of Faraday's law are applied to the second case. We will focus on the third option, where the magnetic field generated by an electrical current is detected by a solid state magnetic sensor. This general scheme can be applied to the measurement of a current driven by a wire or by a conductive strap in a printed circuit board or in an integrated circuit. AC/DC currents can be measured in this way with small, cheap and contact-less systems. Hall devices were for long time the dominant choice for solid state magnetic field sensors. This hegemony was drastically broken in 1988 with the practical realization of giant magnetoresistive (GMR) devices operating at room temperature $[2,3]$.

Three mechanisms have demonstrated to be useful in a major range of applications: permalloy based sensors regarding anisotropic magnetoresistance (AMR), spinvalves (SV) and magnetic tunnel junctions (MTJ) regarding GMR based ones. Their compatibility with standard CMOS technology is in the basis of this rapid growth. In an AMR sensor, the resistance of a permalloy strip depends on the angle between the electric current and the magnetization of the material. A SV consists of two ferromagnetic layers, separated by a nonmagnetic conductor spacer, usually made of $\mathrm{Cu}$. One of these layers has its magnetization pinned, while in the other it is free to rotate. In this case, and due to the low resistance, a current flow parallel to the planes is commonly preferred. An MTJ consists of two ferromagnetic layers, separated by an oxide isolation layer. In this case, the current travels perpendicular to the planes and crosses the isolating barrier by tunnel effect. In any case, an external magnetic field produces a variation of the resistivity [4].

From an electrical point of view, such kind of structures can be perfectly understood as a quadripole with a transfer function relating the output resistance to the input electrical current. MR sensors are commonly arranged in a four elements bridge configuration, due to its inherent linearity and the expected null output in the absence of a magnetic field. In addition, by using a bridge configuration, we could obtain higher output signals which lead to higher sensitivity and also show low temperature dependence.

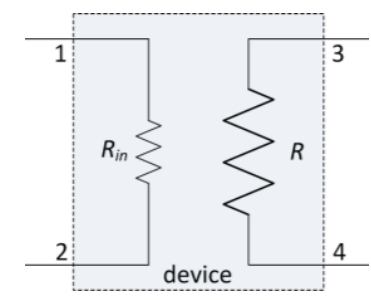

(a)

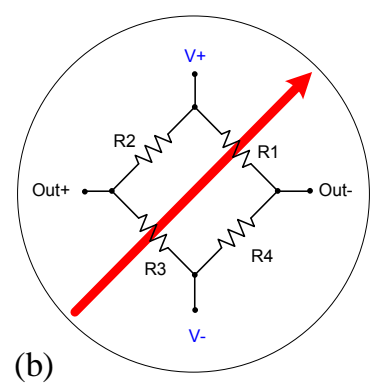

(b)
Fig. 1. (a) magnetoresistive sensing concept, (b) sensor bridge scheme.

Magnetoresistance based sensors have, at least, three intrinsic characteristics that make them potential candidates for electrical current sensors: a high sensitivity (mainly in the case of GMR sensors), a high level of integration (mainly in the case of MTJ sensors) 
Table I. - Sensor characteristics

\begin{tabular}{|l|c|c|c|}
\hline & $\begin{array}{c}\text { ZMC20 } \\
\text { Zetex }\end{array}$ & $\begin{array}{c}\text { HMC1021 } \\
\text { Honeywell }\end{array}$ & $\begin{array}{c}\text { AA004-02 } \\
\text { NVE }\end{array}$ \\
\hline Magnetoresistance & Anisotropic & Anisotropic & Giant (spin-valve) \\
\hline Encapsulation & DIL-14 (modified) & SOIC & SOIC \\
\hline Nominal resistance $(\mathrm{Ohm})$ & $1200-2200$ & $800-1300$ & 5000 \\
\hline Temperature range $\left({ }^{\circ} \mathrm{C}\right)$ & -40 to +120 & -40 to +85 & -50 to +125 \\
\hline Operating range & $\pm 20 \mathrm{~A}$ & $\pm 6 \mathrm{G}$ & $5-35 \mathrm{Oe}$ \\
\hline Sensibility & $0.25 \mathrm{mV} / \mathrm{V} / \mathrm{A}$ & $1.0 \mathrm{mV} / \mathrm{V} / \mathrm{G}$ & $0.9-1.3 \mathrm{mV} / \mathrm{V} / \mathrm{Oe}$ \\
\hline Offset voltage $(\mathrm{mV} / \mathrm{V})$ & \pm 2 & \pm 2 & \pm 4 \\
\hline Bandwidth $(\mathrm{kHz})$ & 100 & 5000 & 1000 \\
\hline Resistive TEMPCO $(\% / \mathrm{K})$ & -0.3 & 0.025 & 0.14 \\
\hline
\end{tabular}

and the possibility of measurement of in-plane magnetic fields (in any case). Additionally, MR current sensors maintain the multiplicative property of Hall sensors, making them promising candidates for direct electrical power measurement. The basic idea of using a MR device as an analog multiplier is very simple, and it's depicted in Fig. 1.
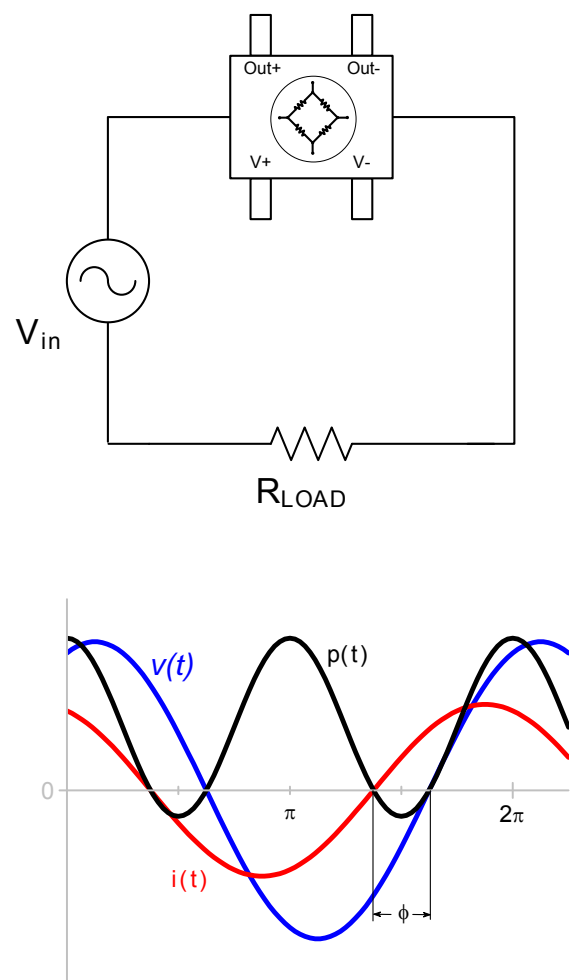

(b)

Fig. 1. (a) Power measurement with a MR sensor,

(b) Description of instantaneous power.

The Wheatstone bridge of the MR sensor is biased by a signal whose value is proportional to the voltage of the measured signal. At the same time, a current proportional to the current of the measured signal is led through a current strap, which generates a magnetic field that influences the Wheatstone bridge. The output (diagonal) voltage of the bridge is (linearly) dependent on the acting magnetic field, and at the same time, it is linearly dependent on the bias voltage. As a direct consequence of these two facts, the output is dependent on the product of the two signals. The idea has been recently applied by using a KMZ51 AMR based commercial sensor [1]. In any case, MR current sensors are on their initial stage in relation to their application in industrial environments, as in the monitoring of power quality [5]. Only few AMR based magnetic sensors can be currently found in the market and, to the best of our knowledge, a unique GMR based one. None of these sensors have an electrical model that can help in the design process of electronic systems based on them.

Because of the particular characteristics of MR devices, the use of a high level language is recommended for the model development. Among current hardware definition languages (HDL), Verilog-A has emerged as a powerful and flexible standard for the analysis and design of analog devices, circuits and systems [6]. In this respect, as far as we know, Verilog-A has not previously applied to the modelling of quasi-linear magneto-resistance structures [7]. In this work, we propose a quasi-static electrical model for general MR based current sensors. For a specific application, the more representative commercial MR sensors are considered.

\section{Sensor characterization and modelling}

A summary of the characteristics of the commercially available MR magnetic field sensors is shown in Tab. I [1]. AA004-2 is a GMR based sensor, and it's not capable of detecting the magnetic field sign. ZMC20 and HMC1021 are based on the AMR effect, and they display a bipolar (magnetic sign detecting) behaviour. All these sensors have a half bridge configuration, with two shielded resistances in order to get an unbalanced response. Even though all of them can be used for current sensing, only ZMC20 is a specific current sensor. HMC1021 and AA004-2 have a SOIC encapsulation, and they should be soldered onto a PCB current track in order to operate as current sensors. On the other hand, ZMC20 has a modified DIL-14 encapsulation, with the current leads integrated on the device. Only ZMC20 current sensor is being considered in the present study.

For DC characterization, a General Purpose Interface Bus (GPIB) controlled setup was used, which included a personal computer, a power supply (PS2521G, Tektronix), a current source (220, Keithley), a data acquisition switch unit (34970A, Agilent) and a multimeter (34401A, Agilent). 
For model parameter extraction purposes, the independent resistance values $\left(R_{1}-R_{4}\right)$ were measured as a function of the driven current, in a given current range. For extracting the exact values, the bridge resistance was measured between consecutive nodes and then $R_{n}$ is calculated from series-parallel considerations. The results are displayed in Fig. 1. From this figure, it can be observed that, for low currents the resistances show a close to linear behaviour. However, for currents higher than $1 \mathrm{~A}$, a cubic trend can be observed. For this reason, the bridge resistances have been modelled making use of the following equation:

$$
R=R_{0}+M R_{1} \cdot I+M R_{2} \cdot I^{2}+M R_{3} \cdot I^{3}
$$

Where $\mathrm{I}$ is the driven current (to be sensed), $R_{0}$ is the value of any bridge resistance for zero current and $M R_{\mathrm{i}}$ are the correspondent polynomial parameters. These parameters have been found by means of curve fitting and the values obtained for the different bridge resistances used are displayed in Tab. II.

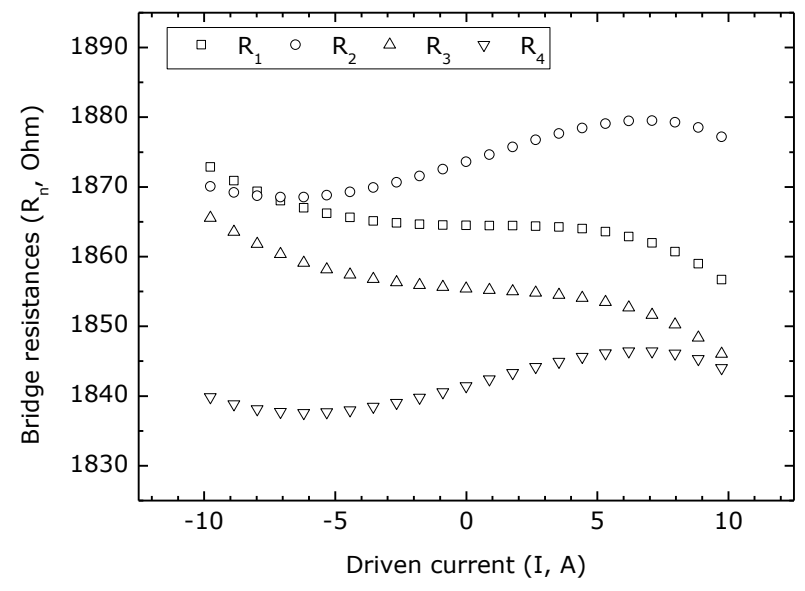

Fig. 1. Bridge resistance versus driven current (ZMC20)

Table II. - ZMC20 bridge magnetic resistances modeling parameters

\begin{tabular}{|l|c|c|c|c|}
\hline & $R_{0}(\Omega)$ & $M R_{1}(\Omega / \mathrm{A})$ & $M R_{2}\left(\Omega / \mathrm{A}^{2}\right)$ & $M R_{3}\left(\Omega / \mathrm{A}^{3}\right)$ \\
\hline $\mathrm{R}_{1}$ & 1870.41 & 1.06 & 0.0055 & -0.0088 \\
\hline $\mathrm{R}_{2}$ & 1879.55 & 0.1666 & 0.0031 & 0.0084 \\
\hline $\mathrm{R}_{3}$ & 1861.31 & 1.24 & 0.0065 & -0.0088 \\
\hline $\mathrm{R}_{4}$ & 1847.32 & 0.0051 & 0.00719 & 0.0083 \\
\hline
\end{tabular}

We can comment on the data shown in Tab. II. Although the values of $R_{1}-R_{4}$ are very similar, they are not exactly the same. These differences produce an output voltage offset that, within this approach, can be taken into account. Moreover, note that $\mathrm{MR}_{1}$ is higher for $R_{1}$ and $R_{3}$ than for $R_{2}$ and $R_{4}$. This fact is due to the half-bridge character of the sensor.

Later on, the model was implemented in Verilog-A with the parameters shown above for the simulation of the Wheatstone bridge sketched in Fig. 2. It can be observed that every resistance is considered as a current-dependant resistance quadripole, and it is modelled individually. Then, they are properly connected in order to build the bridge.

\section{Results and discussion}

The model parameters extracted have been used to simulate the experimental Wheatstone bridge that was characterized in the lab. The voltage response is obtained from the output terminals once the sensors are fed by a direct current ( $\mathrm{I}_{\text {feed }}$ in Fig. 2). The experimental data as well as the simulation results are plotted in Fig. 3 versus the driven current ( $I_{\text {drive }}$ in Fig. 2).

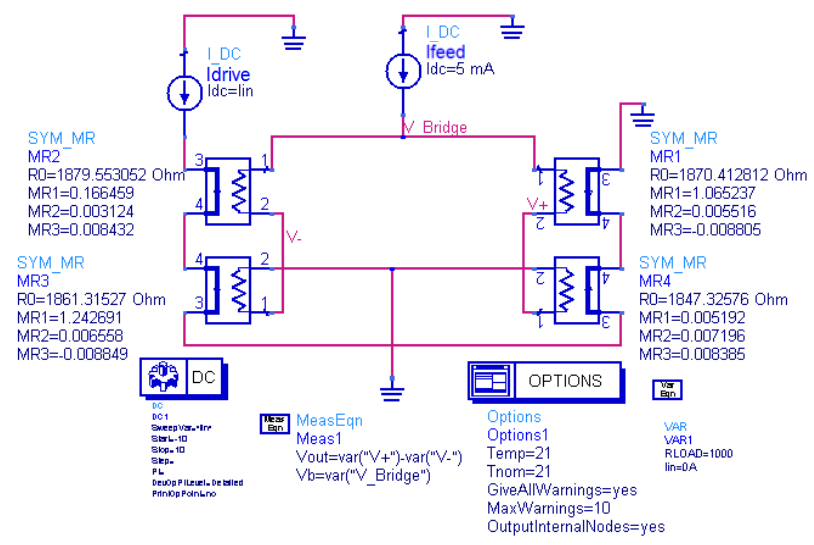

Fig. 2. Wheatstone bridge circuit schematic.

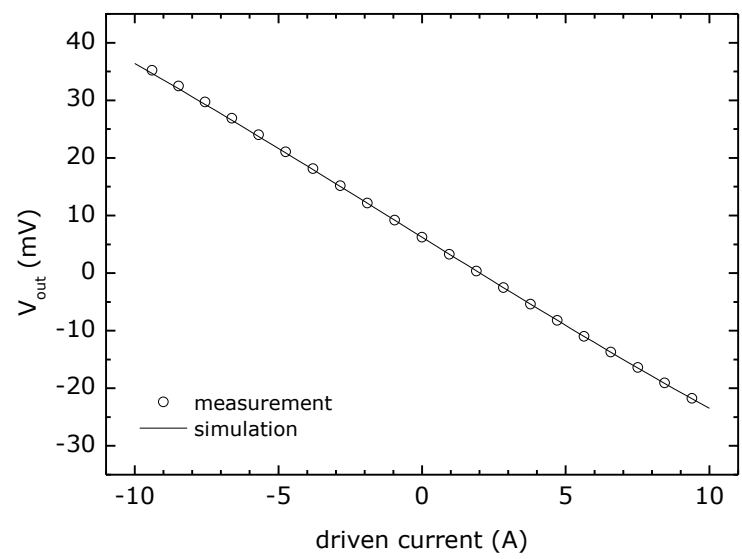

Fig. 3. Output voltage versus driven current for the MR instrumentation bridge

As observed, the simulation results perfectly reproduce the measurements. It's also remarkable the linearization properties of the bridge, which gives a linear response from device nonlinear dependencies. These characteristics, as well as the voltage offset, are reproduced accurately by the model.

\section{Conclusions}

Verilog-A has been successfully used as behavioural modelling language for MR based current sensors. A DC model has been developed and validated by considering experimental measurements in the useful current range of operation. 


\section{Acknowledgement}

Part of the work has been carried out under projects: HP2003/0123 (Ministry of Science and Technology, Spain), GV05/150 (Valencian Regional Government), TIC-3580 (Junta de Andalucía excellence research project) and ENE2008-06588-C04-04 (Ministry of Science and Innovation, Spain and European Regional Development Fund).

\section{References}

[1] C. Reig, M.D. Cubells-Beltrán, D. Ramírez, "GMR based electrical current sensors" in Giant Magnetoresistance: New Research, A.D. Torres and D.A. Pérez, ed. Nova Publishers, 2008, pp. 239-275

[2] M.N. Baibich, J.M. Broto, A. Fert, F.N. van Dau, F. Petroff, P. Eitenne, G. Creuzet, A. Friederich, and J. Chazelas,
"Giant Magnetoresistance of (001)Fe/(001)Cr Magnetic Superlattices", Phys. Rev. Lett. 61 (1988) 2472-2475

[3] G. Binasch, P. Grünberg, F. Saurenbach, and W. Zinn, "Enhanced magnetoresistance in layered magnetic structures with antiferromagnetic interlayer exchange", Phys. Rev. B vol. 39, (1989) 4828-4830

[4] C. Reig, M.D. Cubells-Beltrán, D. Ramírez. "Magnetic Field Sensors Based on Giant Magnetoresistance (GMR) Technology: Applications in Electrical Current Sensing”. Sensors 9 (2009) 7919-7942

[5] C. Reig, D. Ramírez, F. Silva, J. Bernardo, and P. Freitas. "Design, fabrication, and analysis of a spin-valve based current sensor", Sensors and Actuators A-Physical 115 (2004) 259-266.

[6] A.M Roldán, J. B. Roldán, F. Gámiz, "Simulation of CMOS inverters based on the novel surrounding gate transistors. A Verilog-A implementation", MOS-AK 2008/ESSDERC/ESSCIRC, Edimburg, UK, 2008

[7] H. Beltrán, C. Reig, V. Fuster, D. Ramírez, M.D. CubellsBeltrán. "Modeling of Magnetoresistive-Based Electrical Current Sensors: A Technological Approach". IEEE Sensors Journal 7 (2007) 1532-1537 